\title{
SPECTROSCOPIC ANALYSIS OF ECLIPSING SB2 STARS: A CASE STUDY
}

\author{
H. Lehmann ${ }^{1}$, J. Southworth ${ }^{2}$, A. Tkachenko ${ }^{3}$, K. Pavlovski ${ }^{4}$, \\ C. Maceroni ${ }^{5}$ and H. Ak ${ }^{6,7}$
}

\begin{abstract}
The investigation of eclipsing spectroscopic binaries provides basic parameters of stars in a direct way. Whereas the measurable absolute masses can be used to calibrate stellar evolutionary scenarios, the effective temperatures derived from spectroscopic analysis are an important input to light curve and asteroseismic modelling. We compare different methods for investigating eclipsing SB2 stars focusing on radial velocity determination and spectrum decomposition and analysis. Used methods are the two-dimensional cross-correlation technique TODCOR, spectral disentangling with the Fourier transform-based KOREL program, and a grid search-based method of spectrum analysis using spectrum synthesis. The study is based on the investigation of two eclipsing SB2 stars observed by the Kepler satellite mission.
\end{abstract}

\section{Introduction}

The space-based, high-precision photometry by satellite missions like CoRoT and Kepler have opened a new era of accurate measurement of stellar parameters and calibration of evolutionary scenarios. Although both satellites were launched with the primary goal of searching for extrasolar planets, the long-term and almost continuous monitoring of hundreds of thousands of stars has led to the discovery of a large number of variable stars, including about 2100 eclipsing binaries (EBs) in the case of Kepler (Slawson et al. 2011). Among them are binaries with pulsating

\footnotetext{
1 Thüringer Landessternwarte Tautenburg, Germany

2 Keele University, Staffordshire, UK

${ }^{3}$ Instituut voor Sterrenkunde, KU Leuven, Belgium

4 Department of Physics, University of Zagreb, Croatia

5 INAF-Osservatorio Astronomico di Roma, Italy

6 Pennsylvania State University, US

7 Erciyes University, Kayseri, Turkey
} 
components for which asteroseismic analysis can provide insight into the structure of their stellar interiors.

An asteroseismic modelling analysis, on the other hand, requires accurate fundamental parameters of the stars which cannot be gathered from the single, wideband photometry of space missions like Kepler alone. Ground-based multi-colour or spectroscopic follow-up observations are necessary to provide the missing colour information for deriving the temperatures of the stars. In the case of eclipsing SB2 stars, spectroscopy can deliver the radial velocity (RV) curves of the components and so the mass ratio and, in combination with the light curve (LC) analysis, the absolute masses of components.

We applied different techniques to determine the RVs of two eclipsing SB2 stars observed by Kepler and to decompose and analyse the spectra of the components. One of them, KIC 10661783, is an Algol-type system of extremely small mass ratio. The other star, KIC 3858884, is a $\delta$ Scuti pulsator in an EB with highly eccentric orbit. In the following, we compare the results obtained with the different methods and discuss their pros and cons.

\section{KIC 10661783}

\subsection{Motivation}

KIC 10661783 is a short-period $(P \sim 1.23 \mathrm{~d})$ binary star. Southworth et al. $(2011)$ analysed its Kepler LC and found at least 68 frequencies of which 55 can be attributed to pulsation modes of the primary component. The main frequency range lies between 18 and $31 \mathrm{~cd}^{-1}$. The star was suspected to be a so-called oEA star (Mkrtichian et al. 2002), an active Algol-type system where the primary shows $\delta$ Scuti-type oscillations. Our investigation showed, however, that the star is a detached, post Algol-type system, and, it is the Algol with the smallest mass ratio observed so far. Results were published in Lehmann et al. (2013, Paper I).

\subsection{Analysis with KOREL}

KIC 10661783 is a SB2 star with a very faint secondary component. We used the KOREL program (Hadrava 2006) to obtain, from a time series of high-resolution $(R=85000)$ composite spectra taken with the HERMES spectrograph at the Mercator telescope at La Palma, the decomposed spectra of the components.

KOREL is a Fourier transform-based program for spectral disentangling. Spectral disentangling means that the shifts applied to the contributions of the two components to build the decomposed spectra are determined together with optimized orbital parameters. The program also allows computation of the temporal variation in line strengths of the components such as due to eclipses. Drawbacks concern A) the definition of the local continua of the decomposed spectra and B) the normalisation (absolute line depths) of the decomposed spectra.

Problem A) is known as the low-frequency problem in Fourier transform-based methods (e.g., Hensberge et al. 2008) and causes undulations in the continua of 


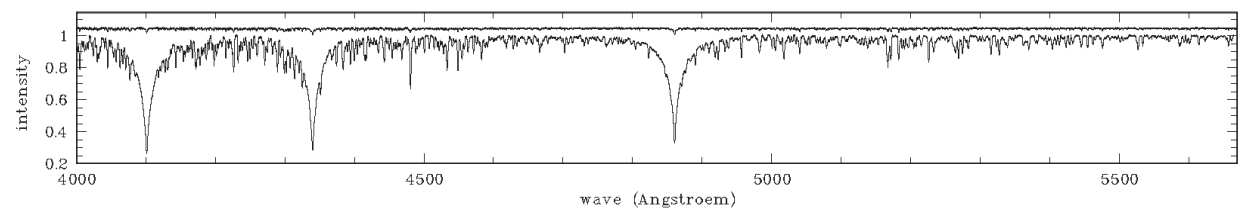

Fig. 1. Decomposed spectra of KIC 10661783 (from Paper I).

Table 1. KIC 10661783: Atmospheric parameters from spectrum analysis.

\begin{tabular}{llcc}
\hline \hline & & primary & secondary \\
\hline$T_{\text {eff }}$ & $(\mathrm{K})$ & $7764 \pm 54$ & $5980 \pm 72$ \\
$\log g$ & $(\mathrm{cgs})$ & 3.9 fixed & 3.6 fixed \\
$v \sin i$ & $\left(\mathrm{~km} \mathrm{~s}^{-1}\right)$ & $79 \pm 4$ & $48 \pm 3$ \\
{$[\mathrm{Fe} / \mathrm{H}]$} & $(\mathrm{dex})$ & $-0.04 \pm 0.07$ & $-0.34 \pm 0.10$ \\
\hline$F_{2} / F_{1}(\lambda=5000 \AA)$ & \multicolumn{2}{c}{$0.067 \pm 0.003$} \\
\hline
\end{tabular}

the decomposed spectra, mainly due to imperfections in the continuum normalisation of the observed spectra. Hensberge et al. (2008) showed that intrinsic light variations can stabilise the separation of the components in the KOREL method. Allowing for variable line strengths in the solution should lift the degeneracy in the determination of the local continua. This approach failed in our case: we ended up with two identical spectra scaled to different line depths. The reason was a wrong weighting of the spectra included, caused by the fact that unusually strong line depths were assigned to few spectra close to eclipse. To overcome this problem, which had not been seen before, we split the spectra into small, overlapping bins and decomposed these bins separately, based on fixed orbital elements determined from a larger wavelength range. Slight undulations in the continua were corrected using spline functions. Figure 1 shows the merged decomposed spectra. It also illustrates the faintness of the companion spectrum.

Problem B) is inherent to all methods of spectrum decomposition. The decomposed spectra are always normalised to the combined continuum flux from both components, as the observed spectra are. The renormalisation to the continuum fluxes of the individual components requires knowledge about the flux ratio between them, e.g., from multi-colour photometry. Since we could not find any such photometry for our star, we tried to solve the problem by including the continuum flux ratio as a free, wavelength-dependent parameter into the analysis of the decomposed spectra. In this case, spectrum analysis has to be done on both components simultaneously. We used the method of spectrum synthesis (Lehmann et al. 2011), defining a $\chi^{2}$ that includes the $\mathrm{O}-\mathrm{C}$ values from the combined fitting of both components (see Paper I). The $\log g$ were fixed to the values obtained from LC analysis.

Table 1 lists the derived atmospheric parameters. We included all parameters into a common error analysis, accounting for all degeneracies between 


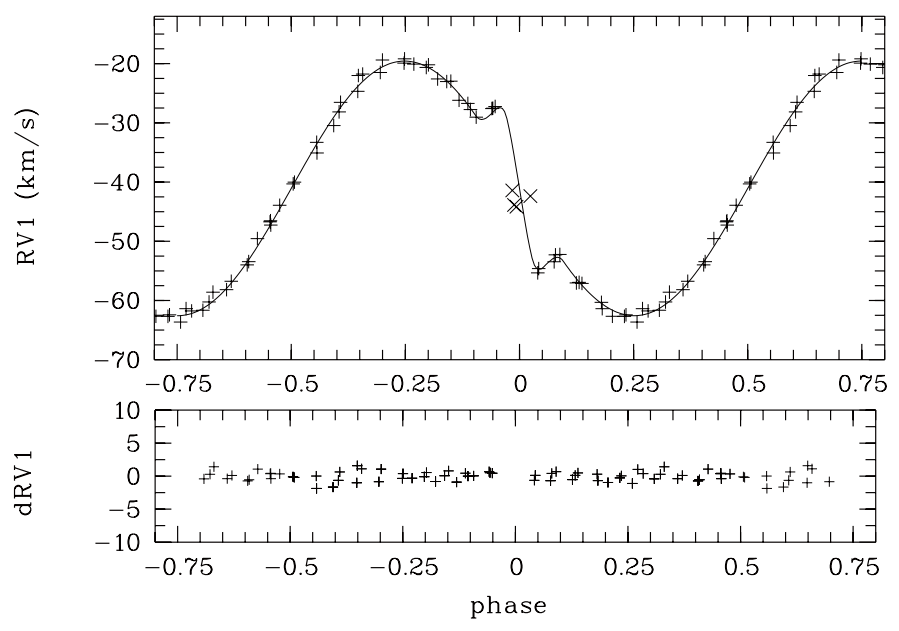

Fig. 2. RVs of the primary component (included RVs are shown by plus signs, outliers by crosses). Solid curve: calculated with PHOEBE. Lower panel: O-C residuals (from Paper I).

the parameters. Table 1 shows that, despite the small flux ratio of 0.067 , the errors of the parameters derived for the two stars are of the same order. The assumed reason is, besides the fact that the lower-temperature companion shows a denser metal lines spectrum, that the errors are mainly determined by the degeneracy between the different parameters, in particular between $T_{\text {eff }}, \log g$, and $[\mathrm{Fe} / \mathrm{H}]$.

KOREL also delivers the orbital parameters (see Table 2 for the most important ones) and the RVs of the components in terms of the shifts applied to the spectra of the components. It does not deliver the errors of the RVs and does not consider any deviations from Keplerian orbital RVs such as caused by the Rossiter-McLaughlin effect (RME, Rossiter 1924; McLaughlin 1924). For that reason, we determined the RVs by a model-independent method using the TODCOR program.

\subsection{Analysis with TODCOR}

TODCOR (Mazeh \& Zucker 1994) performs a two-dimensional cross-correlation between the observed, composite spectra and two different template spectra. It can also determine the flux ratio between the components. We used the best fitting synthetic spectra obtained from spectrum analysis as templates and extended the program to work on a grid of different $v \sin i$ of the templates to count for the different effective line widths due to the RME observed during the eclipses. Finally, we fitted the measured RVs and determined the orbital parameters using the PHOEBE program (Prša \& Zwitter 2005).

Figure 2 shows that the RME can be perfectly fitted which was not the case when using constant $v \sin i$. Figure 3 documents that the flux ratio and effective 
Table 2. Mass ratio and separation based on the KOREL and on the TODCOR RVs.

\begin{tabular}{lcc}
\hline \hline method & $q$ & $a\left(R_{\odot}\right)$ \\
\hline KOREL & $0.0909 \pm 0.0011$ & $6.31 \pm 0.14$ \\
TODCOR & $0.09109 \pm 0.00065$ & $6.370 \pm 0.026$ \\
\hline
\end{tabular}
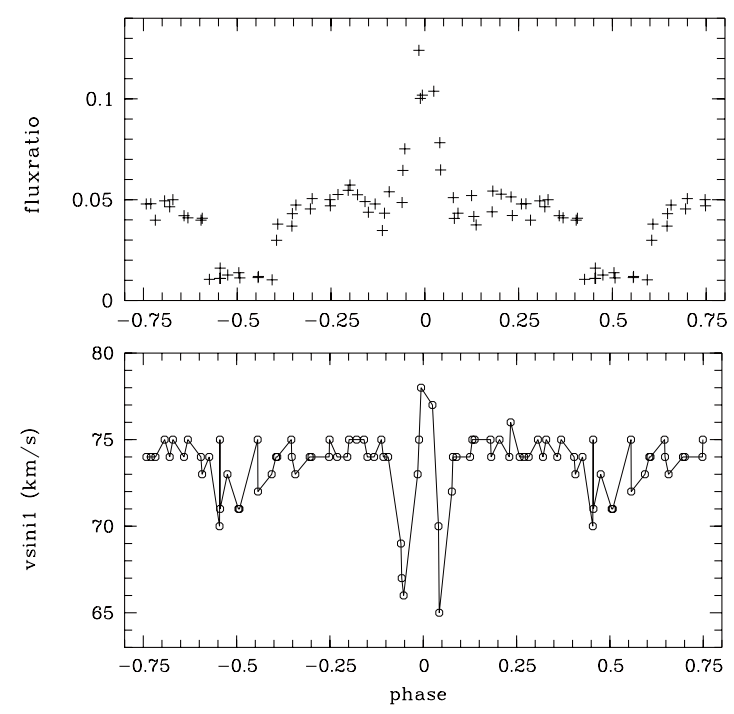

Fig. 3. Flux ratio and $v \sin i$ of the primary folded with the orbital period (from Paper I).

line width $(v \sin i)$ show the expected behaviour. The flux ratio is almost zero during the (total) secondary eclipse and rises during Min I (located at phase zero). The lines get sharper during ingress and egress of Min I and reach their maximum at the centre of the (partial) eclipse. The behaviour during Min II is an artifact due to the fact that Min II is a total eclipse.

Although the usage of variable flux ratio and $v \sin i$ led to a perfect fitting of the RVs during the RME, a closer investigation showed that the absolute values of flux ratio and $v \sin i$ determined with TODCOR are not reliable and are much better constrained by the spectrum analysis. Table 2 compares the two basic parameters mass ratio and semi-major axis based on KOREL and TODCOR RVs. It can be seen that TODCOR plus PHOEBE deliver the more precise values.

\section{KIC 3858884}

\subsection{Motivation}

The Kepler target KIC 3858884 is an EB in a highly eccentric orbit whose LC is characterised by deep eclipses and complex periodic pulsation patterns suggesting 


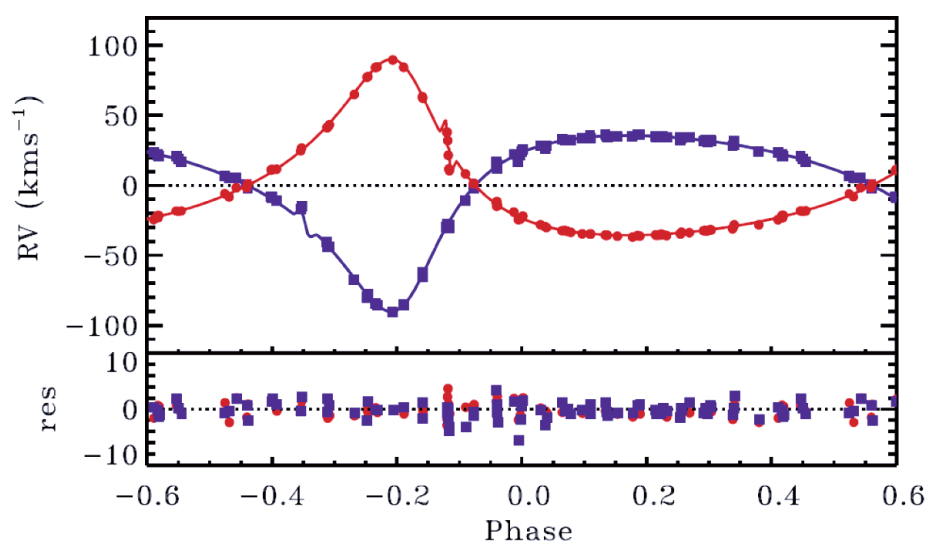

Fig. 4. KOREL-RVs of primary (circles) and secondary (squares), folded with the orbital period. Solid curves: PHOEBE-solutions. Bottom panel: O-C residuals (Paper II).

$\delta$ Scuti pulsations. The pulsation analysis indicates a hybrid nature of the pulsating secondary component. The corresponding high order g-modes might be excited by an intrinsic mechanism or by tidal forces. See Maceroni et al. (2013, Paper II) for the results of a detailed analysis.

\subsection{Analysis with KOREL}

Contrary to KIC 10661783 , KIC 3858884 is an EB consisting of two components of almost the same brightness. For the decomposition with KOREL, we mainly used spectra taken with the 2m-telescope of the Thüringer Landessternwarte Tautenburg $(R=32000)$ and computed two solutions, one by including the spectra taken during Min I and one without these spectra. In the first solution the decomposed spectrum of the primary was broadened by the strong RME (see Fig. 4). The second solution showed undulations in the continua of both decomposed spectra which is due to the fact that no intrinsic light variation occurred anymore. Finally, we found a compromise by excluding all but two spectra taken at Min I. The decomposed spectra did not show any undulations and no difference in the line profiles compared to the exclusion of all spectra around Min I could be found. As before, we added the flux ratio as a free parameter and performed the spectrum analysis on both decomposed spectra simultaneously. The $\log g$ were fixed to the values obtained from the LC analysis. Table 3 lists the results.

\subsection{Analysis with TODCOR}

TODCOR was applied to the observed composite spectra and the measured RVs were fitted with PHOEBE. Both in the TODCOR and in the KOREL RVs, two dominating pulsation frequencies were found after subtracting the orbital solution. The values of these frequencies are in very good agreement with the two main frequencies 
Table 3. KIC 3858884: Atmospheric parameters from spectrum analysis.

\begin{tabular}{llcc}
\hline \hline & & primary & secondary \\
\hline$T_{\text {eff }}$ & $(\mathrm{K})$ & $6810 \pm 70$ & $6890 \pm 80$ \\
$\log g$ & $(\mathrm{cgs})$ & 3.6 fixed & 3.7 fixed \\
$v \sin i$ & $\left(\mathrm{~km} \mathrm{~s}^{-1}\right)$ & $32.2 \pm 1.5$ & $25.7 \pm 1.5$ \\
{$[\mathrm{Fe} / \mathrm{H}]$} & $(\mathrm{dex})$ & $-0.09 \pm 0.07$ & $-0.26 \pm 0.07$ \\
\hline$F_{2} / F_{1}(\lambda=5000 \AA)$ & \multicolumn{2}{c}{$0.904 \pm 0.022$} \\
\hline
\end{tabular}

Table 4. Mass ratio and separation based on the KOREL and on the TODCOR RVs.

\begin{tabular}{lcc}
\hline \hline method & $q$ & $a\left(R_{\odot}\right)$ \\
\hline KOREL & $0.9991 \pm 0.0054$ & $57.08 \pm 0.16$ \\
TODCOR & $0.9880 \pm 0.0068$ & $57.22 \pm 0.20$ \\
\hline
\end{tabular}

found in the Kepler LC. Contrary to the case of KIC 10661783, TODCOR delivered more outliers in RV close to the conjunctions (phases of minimum separation in RV) than KOREL. All KOREL-based RVs could be fitted well by the PHOEBE program. Figure 4 illustrates this showing the KOREL-based orbital solution after subtracting the two mentioned pulsation frequencies. Table 4 compares the mass ratio and separation obtained with the two different methods. It can be seen that the errors from both methods are of the same order.

\section{Conclusions}

We applied different techniques to decompose the spectra of two eclipsing SB2 stars, to analyse their components, and to determine their orbits. Results have been used, in combination with the photometric analysis of the Kepler LCs, to reveal the true nature of KIC 10661783 and to derive basic stellar and system parameters of both targets as a precondition for further asteroseismic modelling (see Papers I and II).

Spectral disentangling with the KOREL program showed that obtaining smooth, undulation-free local continua of the decomposed spectra is not a straightforward task. Although the intrinsic light variation of the EBs during the eclipses should lift the degeneracy in the low-frequency range of Fourier transform, we got no reliable results for KIC 10661783 and had to split the spectra into small wavelength bins using constant line strengths. For KIC 3858884 we got a smooth solution over a large wavelength range when including two well-selected spectra taken during Min I into the disentangling. This was done as a compromise between excluding all eclipse spectra (strong undulations occur) and including all eclipse spectra (the decomposed spectral lines are broadened due to the RME).

The KOREL program delivers RVs in terms of the shifts applied to the contributions of the components to build the decomposed spectra as well as optimised orbital parameters. The solution is constrained by Keplerian motion and does 
not consider any deviations like the RME or proximity effects. TODCOR, on the other hand, delivers model-independent RVs that can be used with some advanced binary code like PHOEBE to model the Keplerian orbit including the RME and proximity effects. In the case of KIC 10661783 where the companion is very faint, the modelling with PHOEBE perfectly fits the RVs derived with TODCOR, including the phases around Min I where the Keplerian curve is strongly distorted by the RME. KOREL, on the other hand, gave no satisfactory RVs at these phases. The situation was inverse when analysing KIC 3858884 that has two components of almost the same brightness. Here, KOREL performed better at the conjunction phases and the RME could also be modelled very well.

In Paper I we introduced an algorithm to derive the flux ratio together with optimised stellar parameters by analysing the two KOREL-decomposed spectra simultaneously, defining a combined $\chi^{2}$. The application to the two target stars showed that we can derive precise flux ratios in this way. But, due to the enlarged number of degrees of freedom, the stellar parameter errors are increased compared to the case when the flux ratio is a priori known and a separate analysis of the (renormalised) spectra is performed.

A necessary precondition for deriving accurate RVs with the TODCOR program during the eclipses was to allow for variable line widths (obviously well approximated by variable $v \sin i$ ). The variations of $v \sin i$ and flux ratio calculated in this way correspond to the expected behaviour but the absolute values derived for the out-of-eclipse phases did not agree with those derived from spectrum analysis. We conclude that TODCOR should not be used to derive accurate stellar parameters by working on a grid of different synthetic templates. As long as the used method for spectrum disentangling does not consider intrinsic deviations from pure Keplerian motion, an independent measurement of RVs using a program like TODCOR is strongly indicated, however.

This work is partly based on observations with the HERMES spectrograph operated at the Mercator telescope at La Palma. We made use of VO-KOREL, provided in the framework of the Czech Virtual Observatory (CZVO) by P. Škoda and J. Fuchs using the Fourier disentangling code KOREL by P. Hadrava. AT is a Postdoctoral Fellow of the Fund for Scientific Research (FWO), Flanders, Belgium.

\section{References}

Hadrava, P., 2006, A\&A, 448, 1149

Hensberge, H., Ilijić, S., \& Torres, K.B.V., 2008, A\&A, 482, 1031

Lehmann, H., Tkachenko, A., Semaan, T., et al., 2011, A\&A, 526, A124

Lehmann, H., Southworth, J., Tkachenko, A., \& Pavlovski, K., 2013, A\&A, 557, A79 (Paper I)

Maceroni, C., Lehmann, H., da Silva, R., et al., 2013, A\&A, submitted (Paper II)

Mazeh, T., \& Zucker, S., 1994, Ap\&SS, 212, 349

McLaughlin, D.B., 1924, ApJ 60, 22 
Mkrtichian, D.E., Kusakin, A.V., Gamarova, A.Yu., \& Nazarenko, V., 2002, ASP Conf. Ser., 259, 96

Prša, A., \& Zwitter, T., 2005, ApJ, 628, 426

Rossiter, R.A., 1924, ApJ, 60, 15

Slawson, R.W., Prša, A., Welsh, W.F., et al., 2011, AJ, 142, 160

Southworth, J., Zima, W., Aerts, C., et al., 2011, MNRAS, 414, 2413 